\title{
Study of logistics distribution route based on improved genetic algorithm and ant colony optimization algorithm
}

\author{
Su Yue ${ }^{1, a}$, Sun $\mathbf{Y i}^{1, b_{*}}$ \\ ${ }^{1}$ Beijing University of Posts and Telecommunications, 100876, China \\ ${ }^{\mathrm{a}}$ suyue@bupt.edu.cn, ${ }^{\mathrm{b} *}$ sunyisse@bupt.edu.cn
}

Keywords: route optimization problem; improved ant colony algorithm; improved genetic algorithm; pheromone;2-OPT sub_routes optimization;

\begin{abstract}
To solve the problem of vehicle routing problem under capacity limitation, this paper puts forward a novel method of logistics distribution route optimization based on genetic algorithm and ant colony optimization algorithm (GA-ACO). On the first stage, improved genetic algorithm with a good global optimization searching ability is used to find the feasible routes quickly. On the second stage, the result of the genetic algorithm is used as the initial solution of the ant colony algorithm to initialize the pheromone. And then improved ant colony optimization algorithm is used to find the optimal solution of logistics distribution route. Experimental results show that the optimal or nearly optimal solutions of the logistic distribution routing can be quickly obtained by this two stages method.
\end{abstract}

\section{Introduction}

For modern logistics enterprises, how to generate vehicle schedules in transportation reasonably, to optimize the transportation line, and to reduce logistics cost has become a core problem of logistics management. Due to the fact that the logistics distribution vehicle routing optimization problem is a Non-deterministic Polynomial Complete problem, using only one method to obtain the global optimal solution is difficult. So some scholars put forward particle swarm optimization combined with genetic algorithm and simulated annealing algorithm combined with genetic algorithm to solve logistics distribution route optimization problem based on the theory of combination optimization[1,2]. Genetic algorithm has the advantages of powerful global search ability and high rates of convergence, but it can't use feedback information which leads to poor search ability, premature convergence and fall into local optimum easily[3].The characteristics of ant colony algorithm is heuristic search and positive feedback mechanism, so it has the advantages of obtaining optimal solution with high efficiency, good local searching ability, distributed computing ability and strong robustness. But due to initial pheromone shortage, the solving speed is slow in early stage of searching[4]. Integrating the advantages of genetic and ant colony algorithm, this paper proposes a two stage method. 


\section{The Design of Logistics Distribution Route Model}

\subsection{Problem Description.}

Logistics distribution route optimization problem is to find the shortest total distance or lowest total freight route of transporting goods from the distribution center to multiple demanding spots by car. And it needs to satisfy the following conditions: (1) Position of each demanding spot and the demand is certain. (2) Each car has a constrain of load capacity. (3) The demand of each demanding spot must be met and be delivered only by one vehicle.

\subsection{Mathematical Model.}

Distribution center represented by 0 delivers goods to demanding spots with amount is $\mathrm{N}$. The demand of $i^{\text {th }}$ demanding spot is $q_{i}$. The distance of demanding spot $i$ and demanding spot $j$ is represented by ${ }^{d_{i, j}}$.(If $i$ or $j$ equals 0 , it represents distribution center.) Distribution center has Num vehicles, each vehicle's load capacity is $\mathrm{W}$ and maximum driving distance is $\mathrm{S}$.

Define the following variables:

$X_{i, j}^{k}=\left\{\begin{array}{ll}0 & \text { The } \mathrm{k}^{\text {th }} \text { car of distribution center doesn't drive from } \mathrm{i} \text { to } \mathrm{j} \\ 1 & \text { The } \mathrm{k}^{\text {th }} \text { car of distribution center drives from } \mathrm{i} \text { to } \mathrm{j}\end{array} Y_{i}^{k}=\left\{\begin{array}{l}0 \text { The demand point } \mathrm{i} \text { isn' } \mathrm{t} \text { distributed by } \mathrm{k}^{\text {th }} \text { car } \\ 1 \text { The demand point } \mathrm{i} \text { is distributed by } \mathrm{k}^{\text {th }} \text { car }\end{array}\right.\right.$

Define the following constraints:

$$
\begin{aligned}
& \sum_{\mathrm{i}=0}^{\mathrm{n}} \sum_{\mathrm{j}=0}^{\mathrm{n}} \mathrm{d}_{i, j} X_{i, j}^{\mathrm{k}} \leq S \quad \mathrm{~K}=1,2,3,4 \ldots \mathrm{Num} ; \\
& \sum_{i=0}^{\mathrm{n}} q_{i} Y_{\mathrm{i}}^{\mathrm{k}} \leq W \\
& \sum_{k=1}^{\mathrm{Num}} Y_{\mathrm{i}}^{\mathrm{k}}= \begin{cases}K & \text { when } \mathrm{i}=0 ; \\
1 & \text { when } \mathrm{i}=1,2, \ldots \mathrm{N} ;\end{cases} \\
& X_{\mathrm{i}, \mathrm{j} . Y_{\mathrm{i}}^{\mathrm{k}}=0 \mid 1 ;}^{\mathrm{k}}
\end{aligned}
$$

Equation 1: A constraint on each vehicle's maximum driving distance

Equation 2: A constraint on each vehicle's maximum load capacity

Equation 3: Constraints of each vehicle must return to distribution center and each demanding spots must be distribute by only one vehicle

On the basis of meet the above constraint conditions minimizing Eq. 5, which represents the minimum path length. This is objective function.

$$
\operatorname{Min}\left(\sum_{\mathrm{k}=1}^{\operatorname{Num}} \sum_{\mathrm{i}=0}^{N} \sum_{\mathrm{j}=0}^{N} \mathrm{~d}_{\mathrm{i}, \mathrm{j}} X_{\mathrm{i}, \mathrm{j}}^{\mathrm{k}}\right)
$$

\section{Algorithm Step}

The existing improved ant colony algorithms mainly put forward some methods for solving ant colony algorithm's problem of easily converging to local optimum, ignoring slow speed caused by initial pheromone shortage in the early searching stage. This paper proposes to use the output of improved genetic algorithm to initialize the pheromone .And making some improvement in 
genetic algorithm and ant colony algorithm for avoiding converging to local optimum.

\subsection{Improved Genetic Algorithm}

1) Decoding

Based on the characteristics of logistics distribution route optimization problem, the author adopts a simple and intuitive natural number coding method[5]. With 0 representing distribution center and $1,2, \ldots, \mathrm{N}$ representing demanding spots. The distribution paths are no more than Num which start and end at distribution center due to no more than Num vehicles in distribution center. In order to reflect the paths of the vehicle distribution in the code, increasing the number of Num-1 virtual distribution centers respectively represented by $\mathrm{Num}+1, \mathrm{Num}+2, \ldots, \mathrm{Num}+\mathrm{N}-1$. Thus, a random arrangement of 1 to $\mathrm{Num}+\mathrm{N}-1$ represents an individual corresponding to a distribution plan.

For example, a distribution center distributes goods to 8 demanding spots with no more than 3 vehicles. 1 to 8 represent demanding spots and 9 to 10 represent virtual distribution centers. A random arrangement of 1 to 10 represents logistics distribution plan.

Table1 Coding scheme example

\begin{tabular}{|l|l|}
\hline individual:2934678 $10{ }^{\prime} 15$ & individual:219367' $10^{\prime} 458$ \\
\hline path1:0-2-0 & path1:0-2-1-0 \\
\hline path2:0-3-4-6-7-8-0 & path2:0-3-6-7-0 \\
\hline path3:0-1-5-0 & path3:0-4-5-8-0 \\
\hline
\end{tabular}

2) Generate initial population

Randomly generating an arrangement of 1 to Num $+\mathrm{N}-1$ forms an individual. In this way, a initial population is generated.

3) Evaluate fitness

The constrains may not be satisfying after steps of genetic operations. So, the fitness function must reflect the feasibility and the cost of corresponding solution.

For individual $r$, the number of unfeasible path in corresponding distribution route scheme is represented by $\mathrm{M}$ (If $\mathrm{M}$ equals 0 , the individual is a feasible solution).Then the fitness function $\mathrm{F}$ is:

$$
\mathrm{F}=1 /(\mathrm{C}+\mathrm{M} * \mathrm{G})
$$

$\mathrm{C}$ is the value of Eq. $5 . \mathrm{G}$ is a punishment weight for each of the unfeasible path, taking a relatively large positive number according to the scope of the objective function.

4)Select

Select optimal and sub-optimal individual measuring by fitness function.

5)Crossover

I adopt a method similar to OX[6] called sequence reversal crossover operator. Compared with other methods, this method can produce a certain degree of variation in the situation of same parents, which has a certain effect on the diversity of the population.

Step A: Determine the location of the exchange area by generating two random number which divide the code into three parts. The code of individual $\mathrm{S} 1=\mathrm{P} 1|\mathrm{P} 2| \mathrm{P} 3$, individual $\mathrm{S} 2=\mathrm{Q} 1|\mathrm{Q} 2| \mathrm{Q} 3$;

Step B: Insert Q2 in front of P2 forming S3' ${ }^{\prime}=\mathrm{P} 1|\mathrm{Q} 2| \mathrm{P} 2 \mid \mathrm{P} 3$. Insert $\mathrm{P} 2$ in front of $\mathrm{Q} 2$ forming $\mathrm{S}^{\prime}{ }^{\prime}=\mathrm{Q} 1|\mathrm{P} 2| \mathrm{Q} 2 \mid \mathrm{Q} 3$.

Step C: Eliminate the same part with Q2 in P1.P2.P3. Eliminate the same part with P2 in Q1.Q2.Q3.

Step D: Reverse the remaining S3' arrangement in addition to the part of Q2, the similar operation on S4'. 
The 2 randomly generated numbers are 4 and 8 :

Table 2 Example of cross

\begin{tabular}{|l|l|}
\hline individual:2934678'10'15 & individual:219367'10'458 \\
\hline Determine exchange area: & Determine exchange area: \\
293|4678|'10'15 & $219\left|367^{\prime} 10^{\prime}\right| 458$ \\
\hline Exchange: & Exchange: \\
293|367'10'|4678|'10'15 & $219|4678| 367^{\prime} 10^{\prime} \mid 458$ \\
\hline Eliminate the same part: & Eliminate the same part: \\
$29|367 ' 10| 48 \mid 15$ & $219|4678| 3^{\prime} 10^{\prime} \mid 5$ \\
\hline Reverse: & Reverse: \\
$51367 ' 10,84 \quad 52$ & $5^{\prime} 10^{\prime} 3 \quad 4678 \quad 91 \quad 2$ \\
\hline
\end{tabular}

6)Mutation

The purpose is to dig out the diversity of individuals in the population, and to overcome the disadvantages of genetic manipulation which may be limited to local solutions.

Mutation is occurred with probability of P. If mutation happens, the exchange number is randomly generated.

If the mutation operation occurs, and the switching frequency $\mathrm{J}=3$;

Table 3 Example of variation scheme

\begin{tabular}{|l|l|l|}
\hline individual: & & 51367'10'8492 \\
\hline Firstly mutation: & location:1.3 & $31567^{\prime} 10^{\prime} 8492$ \\
\hline Secondly mutation: & location:5.8 & 31564'10'8792 \\
\hline Third mutation: & location:6.2 & 3'10'56418792 \\
\hline
\end{tabular}

7) Judge whether the cycle is equal to the maximum number of cycles: If it is ,end up the cycle; If not, return to step (3) and continue to perform.

8) Select the best of the previous $r$ individuals measured by Eq. 5 in population as the initial input of the latter algorithm through decoding.

\subsection{Improved Ant Colony Algorithm}

(1) Initialization

Set initial pheromone. Set the initial $r$ paths of ant colony algorithm by the output of genetic algorithm.Using the Eq. 7 to initial pheromone. Set the maximum iterative algebra and the number of ants k;

$$
\begin{gathered}
\tau_{i, j}=Q_{0}+\Delta \tau_{\mathrm{i}, \mathrm{j}}^{*} \\
\Delta \tau_{\mathrm{i}, \mathrm{j}}^{*}=\sum_{\mathrm{k}=1}^{\mathrm{r}} \Delta \tau_{\mathrm{i}, \mathrm{j}}^{\mathrm{k}}
\end{gathered}
$$

$\Delta \tau_{i, j}^{k}=\left\{\begin{array}{l}0 \text { The path } k \text { doesn't include the route from } i \text { to } j \\ \frac{Q_{0}}{L(k)} \text { The path } k \text { include the route from } i \text { to } j\end{array}\right.$

$Q_{0}$ is a constant. ${ }^{\tau_{i, j}}$ is the concentration of pheromone on the path of $\mathrm{i}$ to $\mathrm{j}$

(2)Tectonic solution

Firstly, according to the Eq. 10 to determine the next point.

The traditional ant colony algorithm based on the probability calculated by Eq. 11 in accordance with the roulette wheel method generates the next access point. The improved algorithm introduces a 
deterministic search and uncertain search. The deterministic search uses the gained experience to guide the path selection, making up for the defects of exploratory search restricted in convergence speed. Through appropriate adjustment of $\mathrm{q}_{1}$, making the deterministic search and exploratory search reasonable collocation can accelerate the convergence speed of ACA.

$$
\begin{aligned}
& \mathrm{d}=\left\{\begin{array}{l}
\text { use roulette wheel method based on formula } 12 \mathrm{q}_{1}>q_{2} \\
\max \left\{\left(\tau_{i, j}\right)^{\alpha}\left(\eta_{i, j}\right)^{\beta}\right\} \mathrm{q}_{1} \leq q_{2}
\end{array}\right. \\
& P_{i, j}^{k}=\frac{\left(\tau_{i, j}\right)^{\alpha}\left(\eta_{\mathrm{i}, j}\right)^{\beta}}{\sum_{\text {s } \in \text { allowed }(k)}\left(\tau_{i, s}\right)^{\alpha}\left(\eta_{\mathrm{i}, \mathrm{s}}\right)^{\beta}} \\
& \eta_{i, j}=\frac{U_{i, j}}{d_{i, j}+C} \\
& U_{\mathrm{ij}}=d_{0, i}+d_{0, j}-d_{i, j}
\end{aligned}
$$

$\mathrm{q}_{1}$ is a constant between 0 to $1 . \mathrm{q}_{2}$ is a random number between 0 to 1 . Allowed(k) is the collection of demanding spots that are not visited. a is the heuristic information coefficient, which indicates the relative importance of trace amount of residual information. The greater the value, the more inclined the ants tend to choose the path of other ants $\beta$ is a parameter to control the influence of visibility. The greater the value, the more close it is to the rule of greedy; $\mathrm{d}_{i, j}$ is the distance from $\mathrm{i}$ to $\mathrm{j}$.

This paper also optimizes the heuristic function $\eta_{\mathrm{i}, j}$. Generally take $\eta_{\mathrm{i}, j}=1 / \mathrm{d}_{i, j}$, but it works poorly when demanding spot $\mathrm{i}$ or $\mathrm{j}$ are also very far away from other spots. The higher of the value of $U_{\mathrm{ij}}$, the directly access to the $\mathrm{j}$ after the visit to $\mathrm{i}$ is more encouraged. A constant $\mathrm{C}$ is added in the denominator to avoid $\eta_{\mathrm{i}, j} \rightarrow \infty$ when $\mathrm{d}_{\mathrm{i}, \mathrm{j}}=0$.

Secondly, determine whether to meet the requirements if access this point: If satisfied, it will be directly added to the current path; If it doesn't satisfied, the ant go back to the distribution center, then start from the distribution center to reach the location. Finally remove the demanding spot from Allowed (k).

Thirdly, judge whether the Allowed $(\mathrm{k})$ is empty:If it is empty, execute 2.4; If not, execute 2.2.

Finally, update local pheromone using Eq. 14

$$
\begin{aligned}
& \tau_{i, j}(t+1)=(1-\lambda) \tau_{i, j}(t)+\lambda \tau_{i, j}^{k} \\
& \tau_{i, j}^{k}=\left\{\begin{array}{l}
0 \text { The path } k \text { doesn't include the route from } i \text { to } j \\
\frac{Q}{L(k)} \text { The path } k \text { include the route from } i \text { to } j
\end{array}\right.
\end{aligned}
$$

(3) By using 2-OPT sub_routes optimization, the solution of each iteration process is improved, the length of the optimal solution is shortened and the rate of convergence algorithm is improved.

Generate two random number i.j. Replace(i.i+1), $(j+1 . j)$ with $(i . j+1),(i+1 . j)$, and after such an exchange path in the line $(i+1, \ldots, j+1)$ is reversed processing. 


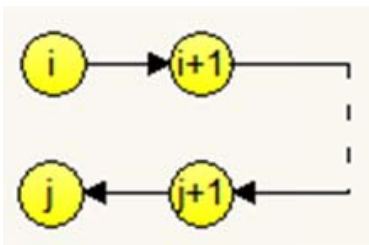

Figure 1 Before

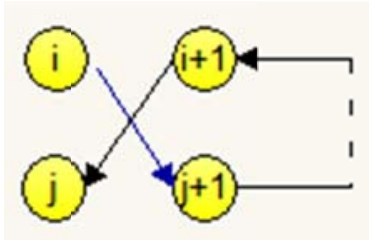

Figure 2 After

(4) Update global pheromone

Choose the better result between initial and handling by 2-OPT sub_routes optimization as the optimal solution of the internal circulation.Using pheromone update rules Eq. 16 to change the pheromone value. When all $\mathrm{K}$ ants construct the solution, one of the shortest paths is the optimal solution of this generation.

$$
\tau_{\mathrm{i}, j}(t+1)=(1-\rho) * \tau_{i, j}(t)+\rho * \Delta \tau_{i, j}(t, t+1)
$$

The adaptive adjustment of the pheromone $\rho$ strategy was improved to avoid the slow convergence speed;

$$
\rho(t+1)=\left\{\begin{array}{l}
\max \left[\delta^{*} \rho(t), \rho_{\min }\right] x=\max \text { non evolutionary algebra } \\
\rho(t) \text { else }
\end{array}\right.
$$

Termination. If the current iteration algebra is more than the setting maximum algebra, then end; Otherwise go to 2) for the next generation of evolution.

\section{Experimental Results and Analysis}

\subsection{Test case}

In this paper, we use the test case in reference [7] and [8]. A logistics center needs to distribute goods to 20 demanding spots with no more than 5 vehicles. The maximum load of the vehicle is $8 \mathrm{~T}$ and the maximum driving distance of a vehicle is $50 \mathrm{~km}$. The coordinate of logistics center is (14.5 $\mathrm{km}, 13 \mathrm{~km}$ ). The coordinates of the 20 demanding spots and the demand for goods is defined in Table 4. Use this algorithm to calculate the problem 10 times, and the results are shown in table 5. Dis in table 5 represents total path distance.

Experimental parameters:

The size of generate initial population and ants' number is $10 ; \alpha=1 ; \beta=2$; The mutation probability is 0.15 ; The maximum iteration number is 500 for both GA and ACO; $\rho_{\min }=0.65$; $\mathrm{q}_{1}=0.55$;Max non evolutionary algebra is 20 ;

\begin{tabular}{|c|c|c|c|c|c|c|c|}
\hline ID & $\mathrm{X}[\mathrm{km}]$ & $\mathrm{Y}[\mathrm{km}]$ & $\mathrm{Q}[\mathrm{t}]$ & ID & $\mathrm{X}[\mathrm{km}]$ & $\mathrm{Y}[\mathrm{km}]$ & $\mathrm{Q}[\mathrm{t}]$ \\
\hline 1 & 12.8 & 8.5 & 0.1 & 11 & 6.7 & 16.9 & 0.9 \\
\hline 2 & 18.4 & 3.4 & 0.4 & 12 & 14.8 & 2.6 & 1.3 \\
\hline 3 & 15.4 & 16.6 & 1.2 & 13 & 1.8 & 8.7 & 1.3 \\
\hline 4 & 18.9 & 15.2 & 1.5 & 14 & 17.1 & 11.0 & 1.9 \\
\hline 5 & 15.5 & 11.6 & 0.8 & 15 & 7.4 & 1.0 & 1.7 \\
\hline 6 & 3.9 & 10.6 & 1.3 & 16 & 0.2 & 2.8 & 1.1 \\
\hline 7 & 10.6 & 7.6 & 1.7 & 17 & 11.9 & 19.8 & 1.5 \\
\hline 8 & 8.6 & 8.4 & 0.6 & 18 & 13.2 & 15.1 & 1.6 \\
\hline 9 & 12.5 & 2.1 & 1.2 & 19 & 6.4 & 5.6 & 1.7 \\
\hline 10 & 13.8 & 5.2 & 0.4 & 20 & 9.6 & 14.8 & 1.5 \\
\hline
\end{tabular}

Table 4 Information about demanding spots 
Table 5 Information about demanding spots

\begin{tabular}{|l|l|l|l|}
\hline Order & Dis $[\mathrm{km}]$ & Order & Dis $[\mathrm{km}]$ \\
\hline 1 & 109.6 & 6 & 107.8 \\
\hline 2 & 110.2 & 7 & 110.4 \\
\hline 3 & 109.1 & 8 & 111.2 \\
\hline 4 & 107.8 & 9 & 107.8 \\
\hline 5 & 111.7 & 10 & 109.1 \\
\hline
\end{tabular}

The average result based on the 10 calculations is $109.47 \mathrm{~km}$ which has a improvement compared with $122.0 \mathrm{~km}$ in reference [7] and $112.5 \mathrm{~km}$ in reference [8]; Besides, the optimal solution is 107.84 $\mathrm{km}$ via this method while the optimal solution in reference [8] is $107.84 \mathrm{~km}$. The paths corresponding to the optimal solution are as follows:

$0-4-3-17-11-20-0 ; \quad 0-8-19-15-16-13-6-0 ; \quad 0-5-14-2-12-9-10-7-1-0 ; \quad 0-18-0$.

\subsection{Analysis}

According to the characteristics of logistics distribution path optimization problem, this paper proposes an optimal path algorithm based on GA-ACO. By introducing crossover and mutation operator, the algorithm can avoid the premature and stagnation of the algorithm in the local search process. The improvements in initial pheromone assigned by genetic algorithm results, the strategy of updating pheromone and on the choice of selecting next demanding spot of ACO enhance the positive feedback effect, thus improving the convergence speed and the global searching ability of the algorithm. The experimental results show that the improved GA-ACO can quickly and effectively obtain the optimal solution or approximate optimal solution. It has a certain reference value for the study of the optimization of logistics distribution routing problem.

\section{Acknowledgements}

This work was financially supported by the Research Innovation Fund for College Students of Beijing University of Posts and Telecommunications(100876).

\section{References}

[1] CHEN Hua-you. Theory and application of combination forecasting method [M]. Beijing: Science Press, 2008

[2] Bischoff C W. The combination of macroeconomic forecasts[J]. Journal of Forecasting, 1989, 8(3): 293-314

[3] LI Shi-yong, LI Pan-chi. A quantum ant colony algorithm for solving continuous space optimization problems [J].Control theory and application, 2008, 25 (2): 237-241

[4] CHEN Wei-dong, WANG Jia. Optimization of logistics distribution route based on hybrid ant colony algorithm [J]. computer engineering and design, 2009, 30 (14): 3383-3385

[5] WANG Hui, REN Chuan-xiang, YIN Chang-chang,Etc. Study on the optimization of logistics distribution route based on Niche Genetic Algorithm[J]. Application Research of computers, 2009.29(10): 2862-2865.

[6] JTJ 026-1-1999, Code for design of ventilation and lighting of highway tunnel[S]

[7] LANG Mao-xiang, HU Si-ji. Study on the optimization of physical distribution routing problem by using hybrid genetic algorithm[J]. Chinese Journal of Management Science, 2002, 10(10): 51-56.

[8] YANG Rui-chen, YUN Qin-xia. Improved ant colony algorithm in mine logistics distribution path[J]. 2004, 28(6): 16-18. 